\section{IDDF2019-ABS-0121 A NOVEL TREATMENT APPROACH IN VISCERAL ARTERY PSEUDOANEURYSM: ENDOSCOPIC ULTRASOUND GUIDED THROMBIN INJECTION}

Sudhir Maharshi* ${ }^{*}$ Deepak Sharma, Shyam Sunder Sharma, Bharat Sapra, Sandeep Nijhawan. Department of Gastroenterology, SMS Hospital, Jaipur, India

10.1136/gutjnl-2019-IDDFabstracts. 158

Background Pseudo aneurysms usually occur after vascular injuries or erosions such as in trauma or inflammation like pancreatitis and are associated with high morbidity and mortality. Digital subtraction angiography with coil embolization is established treatment for aneurysm. There are limited data in the literature on endoscopic ultrasound (EUS) guided thrombin injection for pseudo aneurysm. The aim of the study is to assess the efficacy of EUS guided thrombin injection in pseudo aneurysm.

Methods Prospective data collection was done at SMS Hospital, Jaipur from January 2018 to December 2018. All patients with pseudoaneurysm with a history of gastrointestinal bleed were enrolled consecutively. Data related to demography, laboratory parameters, radiological imaging, pseudo aneurysm and endotherapy were analyzed.

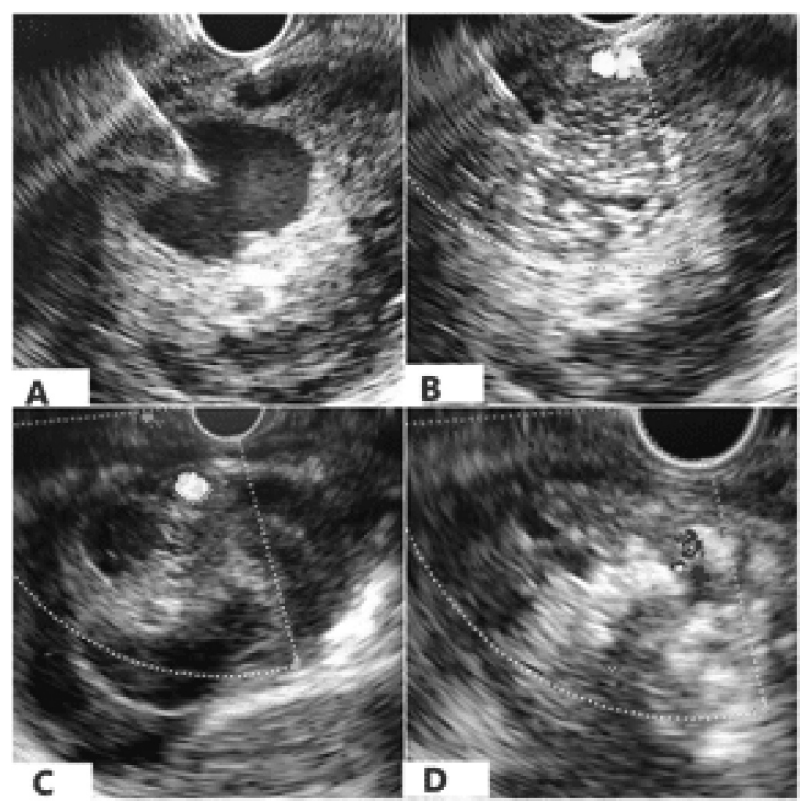

Abstract IDDF2019-ABS-0121 Figure 1 Eus guided thrombin injection in pseudoaneurysm

Results Eight patients with median age 34(27-58) years, all male was studied. Etiology of pseudo aneurysm was chronic pancreatitis in $6(75 \%)$ and idiopathic in $2(25 \%)$ patients. Site of pseudo aneurysm was splenic artery in $5(62.5 \%)$, left hepatic artery in 2(25\%) and gastroduodenal artery in $1(12.5 \%)$ patients. Median size of pseudo aneurysm was $2.9 \mathrm{~cm}$ x 2.6 $\mathrm{cm}(1.8 \times 1.9-4 \times 5 \mathrm{~cm})$. All patients presented with a history of gastrointestinal bleeding and two patients had hemobilia. Median hemoglobin at the time of presentation was $5.2 \mathrm{gm} / \mathrm{dl}$ (4.1-12.2 $\mathrm{gm} / \mathrm{dl})$ and requirement of packed red cell transfusion was 3(0-5) units. Thrombin requirement was 400 IU (200-500IU) for loss of Doppler flow signals. Computed tomography (CT) and EUS after 4 weeks and 3 months showed obliterated pseudoaneurysms in 7 (87.5\%) patients, while recurrence was seen in $1(12.5 \%)$ patient after 6 weeks of thrombin injection. Two patients had post-procedure pain abdomen required intravenous analgesics. (figure 1)

Conclusions EUS-guided thrombin injection provides a new option for the management of pseudoaneurysm.

\section{IDDF2019-ABS-0126 LEVOFLOXACIN SEQUENTIAL THERAPY VERSUS BISMUTH QUADRUPLE THERAPY IN THE SECOND-LINE AND THIRD-LINE TREATMENT OF HELICOBACTER PYLORI -A MULTICENTER RANDOMIZED TRIAL}

${ }^{1}$ Jyh-Ming Liou*, ${ }^{2}$ Po-Yueh Chen, ${ }^{1}$ Chieh-Chang Chen, ${ }^{3}$ Yu-Jen Fang, ${ }^{4}$ Ming-Jong Bair, ${ }^{1} J a w-T o w n$ Lin, ${ }^{1}$ Ming-Shiang Wu. ${ }^{1}$ National Taiwan University Hospital, Taiwan; ${ }^{2}$ Department of Internal Medicine, Chia-Yi Christian Hospital, Taiwan; ${ }^{3}$ Department of Internal Medicine, National Taiwan University Hospital, Yun-Lin Branch, Taiwan; ${ }^{4}$ Department of Internal Medicine, Mackay Memorial Hospital, Taitung branch, Taiwan

\subsection{6/gutjnl-2019-IDDFabstracts.159}

Background We aimed to compare the efficacy and safety of 14-day levofloxacin sequential therapy versus 10 -day bismuth quadruple therapy in the second-line and third-line treatment of Helicobacter pylori (H. pylori) infection.

Methods $H$. pylori infected patients who failed after one treatment were eligible in this open-labeled, multicenter, randomized trial, and were randomized to receive (1) levofloxacin sequential therapy (EAML): esomeprazole $40 \mathrm{mg}$ and amoxicillin $1 \mathrm{~g}$ for the first 7 days, followed by esomeprazole 40 $\mathrm{mg}$, metronidazole $500 \mathrm{mg}$, and levofloxacin $250 \mathrm{mg}$ for another 7 days (all twice daily); or (2) bismuth quadruple therapy (BQ): esomeprazole $40 \mathrm{mg}$ twice daily, bismuth tripotassium dicitrate $300 \mathrm{mg}$ four times a day, tetracycline 500 $\mathrm{mg}$ four times a day, and metronidazole $500 \mathrm{mg}$ three times a day, for 10 days. The primary end point was the eradication rate in the second-line treatment according to intention to treat (ITT) analysis. The minimum inhibitory concentrations were determined by agar dilution test.

Results A total of 560 patients have been recruited and results were available for analysis in 533 patients up to Jan 2019. The demographic characteristics and antibiotic resistance rates were similar across the two treatment groups. The eradication rate in the second line treatment was $88.3 \%(235 / 266)$ and $88.4 \%$ $(236 / 267)$ in the levofloxacin sequential therapy and bismuth quadruple therapy groups, respectively $(p=1.000)$ in the ITT analysis. The eradication rates were $89.7 \%$ (235/262) and $92.9 \%(236 / 254)$ in the levofloxacin sequential therapy and bismuth quadruple therapy according to PP analyses, respectively $(p=0.195)$. The efficacy of levofloxacin sequential therapy, but not bismuth quadruple therapy, appeared to be affected by levofloxacin resistance. The frequency of any adverse effects was higher in patients treated with bismuth quadruple therapy than levofloxacin sequential therapy $(76.4 \%$ vs. $44.1 \%, \mathrm{p}<0.001)$. The efficacy of bismuth quadruple therapy and levofloxacin sequential therapy in the third-line treatment were $70.6 \%(12 /$ $17)$ and $63.6 \%(7 / 11)$, respectively. (table 1) 